ITEP-TH-35/02

TPI-MINN-02/14

UMN-TH-2101/02

\title{
Testing Nonperturbative Orbifold Conjecture
}

\author{
A. Gorsky \\ Institute of Theoretical and Experimental Physics, B.Cheremushkinskaya 25, \\ Moscow, 117259, Russia \\ M. Shifman \\ Theoretical Physics Institute, University of Minnesota, 116 Church St. S.E., \\ Minneapolis, MN 55455, USA
}

\begin{abstract}
We discuss Strassler's hypothesis of matching nonperturbative effects in orbifold pairs of gauge theories which are perturbatively planar equivalent. One of the examples considered is the parent $\mathcal{N}=1 \mathrm{SU}(N)$ supersymmetric Yang-Mills theory and its nonsupersymmetric orbifold daughter. We apply two strategies allowing us to study nonperturbative effects: (i) low-energy theorems; (ii) putting the theory on small-size $T^{4}$ or $R^{3} \times S^{1}$. Then both the parent and daughter theories are weakly coupled and amenable to quasiclassical treatment. In all cases our consideration yields a mismatch between the parent and daughter theories. Thus, regretfully, we present evidence against Strassler's hypothesis. We discuss in passing a brane picture related to our consideration.
\end{abstract}




\section{Introduction}

In spite of impressive progress in description of nonperturbative results in the supersymmetric (SUSY) gauge theories during the last decade the progress in non-SUSY gauge theories is quite modest. That is why any tool invented to connect SUSY and non-SUSY theories in a controllable way gives rise to new hopes. Among recent promising advances is the orbifolding procedure introduced in the string theory context [1, 2] (with the purpose of reducing the amount of SUSY). It was shown that both parent and daughter theories enjoy the same planar limit.

Later on, a similar procedure was worked out in field theory: it was proved that the parent and daughter theories have the same perturbative behavior at large $N$ (i.e. for all planar graphs) provided a proper rescaling of the coupling constant is made [3, 4, 5] (see also [6] for an earlier approach).

At the nonperturbative level so far next to nothing is known. It was shown in [7], starting from the brane picture of the orbifold theory, that the correspondence survives at the nonperturbative level in a limited sense - for the low-energy effective actions - if both parent $(\mathcal{N}=2)$ and daughter $(\mathcal{N}=1)$ theories are supersymmetric. In this case the Riemann surfaces governing the low-energy behaviors coincide after the above-mentioned rescaling of the coupling constant. Further attempts to consider the orbifoldization at the nonperturbative level in the theory with fundamental matter have been made in [8].

In a recent paper [9] Strassler put forward a bold and beautiful conjecture that the correspondence between the planar perturbative limits between the parent/daughter theories is an exact property and is valid including all nonperturbative effects at large $N$ under an appropriate rescaling of coupling constants. More exactly, Strassler's nonperturbative orbifold (NPO) conjecture reads: "correlation functions calculated in those vacua which appear in both (parent/daughter) theories, for the operators which appear in both theories, are identical." This implies, in particular, the coincidence of the corresponding spectral functions. If information on the spectral function in the parent theory is available, the NPO conjecture allows one to predict that in the daughter theory.

The NPO conjecture leads to remarkable consequences [9] if the parent theory is $(\mathcal{N}=1)$ supersymmetric gluodynamics (then the daughter one is not supersymmetric). For instance, were the NPO conjecture true it would give rise to infinite degeneracies between the bosonic states of the daughter theory which the latter would inherit from the parent one. In short, the NPO conjecture could become a powerful tool in the studies of non-Abelian gauge theories, a major finding in this range of questions.

In this paper we suggest simple tests of Strassler's conjecture. To our deep regret we observe that these tests do not support the NPO conjecture - in all cases where we could quantitatively compare nonperturbative effects there is a mismatch between the parent and daughter theories.

What do we know for certain of nonperturbative aspects in non-Abelian gauge 
theories? First, at strong coupling, there exist exact low-energy theorems. One can confront the NPO conjecture with these theorems.

Secondly, one can make non-Abelian gauge theories weakly coupled by considering special geometries of the world sheet. At weak coupling nonperturbative effects can be reliably analyzed using quasiclassical methods. Since the correspondence between the parent and the orbifold theory is the property of the planar graphs [3, 4, 5], it does not depend on geometry of the world sheet. Instead of $R^{4}$ one can consider $T^{4}$ or $R^{3} \times S^{1}$. If the size of the compact dimension(s) is small compared to $\Lambda^{-1}$ ( $\Lambda$ is the scale parameter of the gauge theory), of the gauge theory,

$$
\Lambda \sim M_{\mathrm{uv}} \exp \left(-\frac{8 \pi^{2}}{3 k N g_{P}^{2}}\right)=M_{\mathrm{uv}} \exp \left(-\frac{8 \pi^{2}}{3 N g_{D}^{2}}\right)
$$

the gauge theory never becomes strongly coupled. (Here $g_{P}$ and $g_{D}$ are the gauge couplings in the parent and daughter theories, respectively, $k$ is the orbifoldization degree, $M_{\mathrm{uv}}$ is the ultraviolet cut-off.) At weak coupling the theory is under reliable theoretical control and one can compare corresponding nonperturbative effects in the parent and orbifold theories.

In this paper we focus on these two sets of tests. At strong coupling we analyze low-energy theorems for topological susceptibilities and patterns of chiral symmetry breaking. We observe non-matching of certain factors related to $k$, the degree of orbifoldization (Secs. 3.2 and 4). Then we consider parent/daughter theories on $T^{4}$ and $R^{3} \times S^{1}$, at weak coupling. In the first case one deals with torons (Sec. 3.3), in the second with "monopole instantons" (Sec. 3.4). In both cases there is a clear-cut mismatch between the numbers of the fermion zero modes, and, hence, the corresponding condensates.

Thus, the overall conclusion of our investigation is, unfortunately, negative the evidence presented suggests that the perturbative planar equivalence does not extend at nonperturbative level.

\section{General remarks}

Instantons, historically the first example of nonperturbative field configuration [10 in Yang-Mills, are irrelevant for our purposes since the instanton action scales as $8 \pi^{2} / g^{2} \sim N$ at large $N$. Hence, instanton contributions die off as $\exp (-N)$. We have to consider nonperturbative fields configurations with action scaling as $8 \pi^{2} /\left(N g^{2}\right)$. Such configurations are known in non-Abelian gauge theories on $T^{4}$ and $R^{3} \times S^{1}$.

For $Z_{k}$ orbifoldization (see Sec. 3) the gauge invariant chiral operator of the lowest dimension one can build of the chiral fermion fields in the daughter theory has the structure

$$
\mathcal{O}_{k}=\prod_{\ell=1}^{k} \chi_{\ell, \ell+1}
$$


where the color and Lorentz indices are suppressed. The color indices must be convoluted cyclically, while the Lorentz ones can be convoluted in an arbitrary way. One more class of gauge invariant operators is represented by baryons

$$
B_{l}=\operatorname{det}\left(\chi_{\ell, \ell+1}\right) \text {. }
$$

The cases of even and odd $k$ should be treated separately. If $k$ is even, then the operator $\mathcal{O}_{k}$ in Eq. (2) is bosonic. At $k=2$ it is the orbifold projection of the gluino operator $\lambda \lambda$ of the parent theory. At $k=4,6, \ldots$ the operator $\mathcal{O}_{k}$ can be obtained as the orbifold projection of $(\lambda \lambda)^{k / 2}$. The vacuum expectation value of $(\lambda \lambda)^{k / 2}$ in the parent theory is saturated by the gauge field configuration with the topological charge $k / 2$ times the minimal one. We will be interested mostly in the case $k=2$. In this case the operators (2) and $\lambda \lambda$ can be used as mass terms. Limiting oneself to $k=2$ is sufficient to show that the NPO conjecture does not work.

If $k$ is odd, then the operator $\mathcal{O}_{k}$ in Eq. (2) is fermionic. It is the orbifold projection of the parent theory operator of the type $\lambda^{k}$. Needless to say that neither $\mathcal{O}_{k}$ nor $\lambda^{k}$ can have vacuum expectation values. In this case one may confront the correlation function $\left\langle\lambda^{k}(x) \lambda^{k}(y)\right\rangle$ in the parent theory with $\left\langle\mathcal{O}_{k}(x) \mathcal{O}_{k}(y)\right\rangle$ in the daughter one. The gauge field configuration saturating $\left\langle\lambda^{k}(x) \lambda^{k}(y)\right\rangle$ has the topological charge $k$ times the minimal one.

\section{Supersymmetric gluodynamics and its daugh- ter theory}

Let the parent theory be $\mathcal{N}=1$ SUSY Yang-Mills theory with $\mathrm{SU}(k N)$ gauge group, $k$ is an integer,

$$
\mathcal{L}=\frac{1}{g_{P}^{2}}\left(-\frac{1}{4} F_{\mu \nu}^{a} F^{\mu \nu, a}+i \bar{\lambda}_{\dot{\alpha}}^{a} \mathcal{D}^{\dot{\alpha} \alpha} \lambda_{\alpha}^{a}\right),
$$

where $\lambda_{\alpha}$ is the Weyl spinor in the adjoint. This theory has the classical global $\mathrm{U}(1)_{R}$ symmetry which is broken quantum-mechanically by anomaly down to $Z_{2 k N}$. The theory has $k N$ chirally asymmetric vacua labeled by the value of the gluino condensate $\langle\lambda \lambda\rangle$.

The orbifold projection eliminates the gluon fields with the color indices outside $N \times N$ blocks on the diagonal; it also eliminates the gluino fields with the indices outside $N \times N$ blocks above the diagonal (see Fig. 1). Hence, the daughter theory is the gauge theory with $(\mathrm{SU}(N))^{k}$ gauge group and $k$ bifundamentals. The Lagrangian of the daughter theory is

$$
\mathcal{L}=\frac{1}{g_{D}^{2}}\left(-\frac{1}{4} \sum_{\ell=1}^{k} F_{(\ell) \mu \nu} F_{(\ell)}^{\mu \nu}+i \sum_{\ell=1}^{k} \bar{\chi}_{\ell, \ell+1} \mathcal{D} \chi_{\ell, \ell+1}\right), \quad \chi_{k, k+1} \equiv \chi_{k, 1},
$$

where the covariant derivative is defined as $D_{\mu}=\partial_{\mu}-i \sum_{\ell} A_{(\ell) \mu} T^{(\ell)}$, while $T^{(\ell)}$ are the generators of the gauge symmetry with respect to the group $\mathrm{SU}(N)$ number $\ell$. 
The fermion fields are denoted by $\chi_{\ell, \ell+1}$. The first subscript belongs to fundamental, the second to antifundamental. The coupling constants are subject to the relation

$$
g_{D}^{2}=k g_{P}^{2} .
$$

The daughter theory is non-supersymmetric. It is expected [9] to have $N$ discrete vacua.

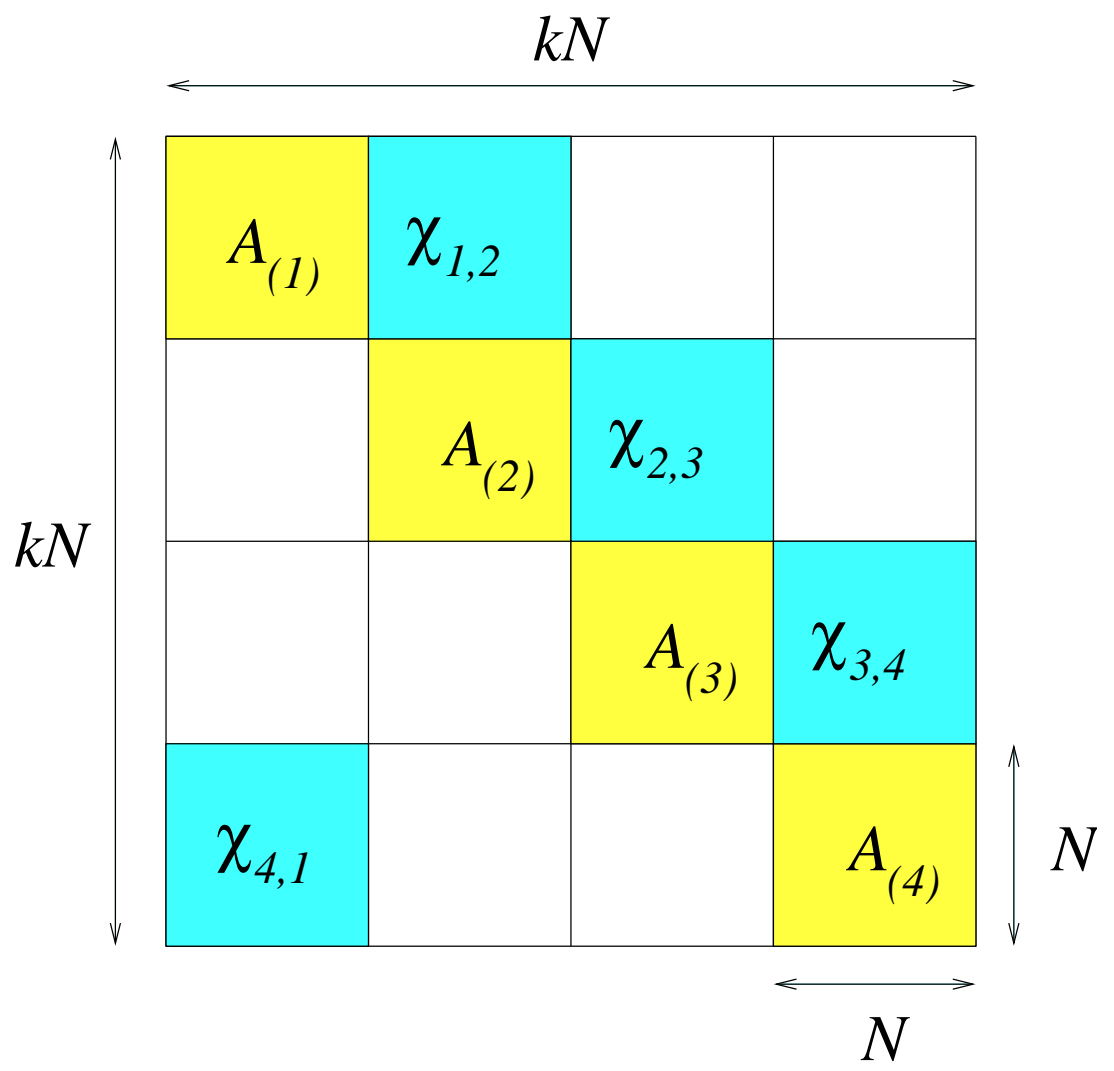

Figure 1: The field contents of the daughter theory upon $Z_{k}$ orbifoldization. The example shown corresponds to $Z_{4}$ orbifoldization. The gauge fields are denoted by $A_{(\ell)}$ while the fermion fields are denoted by $\chi_{\ell, \ell+1}$. The first subscript belongs to fundamental, the second to antifundamental.

In particular, if $k=2$, the daughter theory has the gauge symmetry $\mathrm{SU}(N) \times$ $\mathrm{SU}(N)$, with the matter sector consisting of two Weyl spinors - one of them is $\{N, \bar{N}\}$ while the other $\{\bar{N}, N\}$; these two Weyl spinors can be combined into one bifundamental Dirac spinor. According to the NPO conjecture, a possible correspondence between the theories (困) and (50) extends to those operators which exist in the parent theory and have non-trivial projections in the daughter one, i.e. (gauge invariant) operators invariant under the action of $Z_{k}$. At $k=2$, as an example, one can consider the operator $\lambda \lambda$ and its projection $\chi_{1,2} \chi_{2,1}$, and correlation functions 
of the type

$$
\langle\lambda \lambda(0), \bar{\lambda} \bar{\lambda}(x)\rangle \leftrightarrow\left\langle\chi_{1,2} \chi_{2,1}(0), \bar{\chi}_{1,2} \bar{\chi}_{2,1}(x)\right\rangle .
$$

For arbitrary values of $k$ bilinear in $\chi$ operators are not gauge invariant; therefore, the correspondence pairs are

$$
\underbrace{\lambda \ldots \lambda}_{k} \leftrightarrow \prod_{\ell=1}^{k} \chi_{\ell, \ell+1},
$$

where the color indices in $\lambda^{k}$ are convoluted cyclically. For even $k$ these operators are bosonic while for odd $k$ fermionic.

In what follows we will derive low-energy theorems for theories formulated on $R 4$ (in strong coupling) and, alternatively, analyze the parent and daughter theories on either $T^{4}$ or $R^{3} \times S^{1}$, with a small size of the compact dimension (in weak coupling). For both purposes we need to know chiral anomalies.

\subsection{Divergence of the axial current}

The chiral currents in the parent and daughter theories are

$$
\begin{aligned}
J_{\mu}^{P} & =\frac{1}{2}\left(\sigma_{\mu}\right)_{\alpha \dot{\alpha}} J_{P}^{\alpha \dot{\alpha}} \equiv-\frac{1}{g_{P}^{2}} \lambda^{a} \sigma_{\mu} \bar{\lambda}^{a}, \\
J_{\mu}^{D} & =\frac{1}{2}\left(\sigma_{\mu}\right)_{\alpha \dot{\alpha}} J_{D}^{\alpha \dot{\alpha}} \equiv-\frac{1}{g_{D}^{2}} \sum_{\ell=1}^{k} \chi_{\ell, \ell+1} \sigma_{\mu} \bar{\chi}_{\ell, \ell+1},
\end{aligned}
$$

where the sub(super)scripts $P$ and $D$ refer to the parent and daughter theories, respectively. The chiral anomalies have the form

$$
\begin{aligned}
\partial^{\mu} J_{\mu}^{P} & =\frac{k N}{16 \pi^{2}} F_{\mu \nu}^{a} \tilde{F}_{\mu \nu}^{a}, \\
\partial^{\mu} J_{\mu}^{D} & =\frac{N}{16 \pi^{2}} \sum_{\ell=1}^{k} F_{\mu \nu}^{a_{\ell}} \tilde{F}_{\mu \nu}^{a_{\ell}},
\end{aligned}
$$

where the color index $a$ in Eq. (11) covers $\mathrm{SU}(k N)$ while $a_{\ell}$ in Eq. (12) the $\ell$-th $\mathrm{SU}(N)$ factor. Let us recall that, with our normalization, the topological charge

$$
Q=\left(32 \pi^{2}\right)^{-1} \int d^{4} x F_{\mu \nu}^{a} \tilde{F}_{\mu \nu}^{a} .
$$

At $k>2$ the daughter theory admits no mass term for the fermion field. However, at $k=2$ a mass term is possible. It has a counterpart in the parent theory - a gluino mass term - which breaks supersymmetry. We introduce these mass terms, with a small mass parameter $m$, with the intention to derive a low-energy theorem to leading order in $m$. More specifically, in the parent theory

$$
\Delta \mathcal{L}_{m}=-\frac{1}{2} \frac{m}{g_{P}^{2}}\left(\lambda^{a} \lambda^{a}+\bar{\lambda}^{a} \bar{\lambda}^{a}\right)
$$


while in the daughter one

$$
\Delta \mathcal{L}_{m}=-\frac{m}{g_{D}^{2}}\left(\chi_{1,2} \chi_{2,1}+\bar{\chi}_{1,2} \bar{\chi}_{2,1}\right)
$$

The operator (15) is the orbifold projection of (14). In both cases the mass terms are normalized in such a way that the free fermion propagator has the standard form $(\not p-m)^{-1}$.

If the mass terms are included, the divergence of the currents (9) and (10), besides the anomalous part presented in Eqs. (11) and (12), has a nonanomalous (classical) part,

$$
\begin{aligned}
\partial^{\mu} J_{\mu}^{\mathrm{P}} & =\frac{2 N}{16 \pi^{2}} F_{\mu \nu}^{a} \tilde{F}_{\mu \nu}^{a}+i \frac{m}{g_{P}^{2}}\left(\lambda^{a} \lambda^{a}-\bar{\lambda}^{a} \bar{\lambda}^{a}\right) \\
\partial^{\mu} J_{\mu}^{\mathrm{D}} & =\frac{N}{16 \pi^{2}} \sum_{\ell=1}^{2} F_{\mu \nu}^{a_{\ell}} \tilde{F}_{\mu \nu}^{a_{\ell}}+2 i \frac{m}{g_{D}^{2}}\left(\chi_{1,2} \chi_{2,1}-\bar{\chi}_{1,2} \bar{\chi}_{2,1}\right) .
\end{aligned}
$$

\subsection{Low-energy theorem for topological susceptibilities}

The topological susceptibility reflects dependence of the vacuum energy on the vacuum angle $\theta$. For massless fermions such dependence is absent and the topological susceptibility vanishes. However, if $m \neq 0$, the topological susceptibility does not

vanish and can be readily derived to leading order in $m$. In the parent theory one defines it as follows

$$
\mathcal{T}_{P}=i \int d^{4} x\left\langle\frac{1}{32 \pi^{2}} F_{\mu \nu}^{a} \tilde{F}_{\mu \nu}^{a}(x), \frac{1}{32 \pi^{2}} F_{\mu \nu}^{a} \tilde{F}_{\mu \nu}^{a}(0)\right\rangle .
$$

In the daughter theory there are two vacuum angles. We are interested in the vacuum response to a $Z_{2}$ invariant combination (remember, for the time being we consider $Z_{2}$ orbifoldization), namely

$$
\mathcal{T}_{D}=i \frac{1}{2} \int d^{4} x\left\langle\frac{1}{32 \pi^{2}} \sum_{\ell=1}^{2} F_{\mu \nu}^{a_{\ell}} \tilde{F}_{\mu \nu}^{a_{\ell}}(x), \frac{1}{32 \pi^{2}} \sum_{\ell=1}^{2} F_{\mu \nu}^{a_{\ell}} \tilde{F}_{\mu \nu}^{a_{\ell}}(0)\right\rangle .
$$

The overall factor $1 / 2$ in Eq. (19) is introduced to provide proper normalization: with this factor included the planar perturbative expansion of the correlation functions $\int d^{4} x \exp (i q x)\langle F \tilde{F}(x), F \tilde{F}(0)\rangle$ at large momentum transfer will be the same in the parent and daughter theories.

Using Eqs. (16) and (17) and the fact that both theories have no massless particles in the $m \rightarrow 0$ limit, it is easy to obtain

$$
\begin{aligned}
& \mathcal{T}_{P}=\frac{1}{8 N^{2}} \frac{m}{g_{P}^{2}}\left\langle\lambda^{a} \lambda^{a}+\bar{\lambda}^{a} \bar{\lambda}^{a}\right\rangle, \\
& \mathcal{T}_{D}=\frac{1}{2 N^{2}} \frac{m}{g_{D}^{2}}\left\langle\chi_{1,2} \chi_{2,1}+\bar{\chi}_{1,2} \bar{\chi}_{2,1}\right\rangle,
\end{aligned}
$$


where terms $O\left(\mathrm{~m}^{2}\right)$ are omitted. In accordance with the NPO hypothesis, the condensates on the right-hand side must be calculated in the vacuum state which is present in both theories. One can normalize them as follows: to leading order in $m$ the vacuum energy densities $\mathcal{E}$ are ${ }^{1}$

$$
\mathcal{E}_{P}=\frac{1}{2} \frac{m}{g_{P}^{2}}\left\langle\lambda^{a} \lambda^{a}+\bar{\lambda}^{a} \bar{\lambda}^{a}\right\rangle, \quad \mathcal{E}_{D}=\frac{m}{g_{D}^{2}}\left\langle\chi_{1,2} \chi_{2,1}+\bar{\chi}_{1,2} \bar{\chi}_{2,1}\right\rangle
$$

where the condensates $\lambda \lambda$ and $\chi_{1,2} \chi_{2,1}$ are $O\left(m^{0}\right)$. Demanding the equality of the energy densities (i.e. $\mathcal{E}_{P}=\mathcal{E}_{D}$ ) we see that the topological susceptibilities differ by a factor of 2 :

$$
\mathcal{T}_{P}=\frac{1}{2} \mathcal{T}_{D}
$$

This difference reflects the fact that the numbers of vacuum states in the parent and daughter theories are different, and although we deal with the expectation values in the given vacuum, they still do "remember" of this difference.

\subsection{Weak coupling: theory on $T^{4}$}

Instead of $R^{4}$ let us take $T^{4}$ as the world volume. Topologically nontrivial gauge fields produce nonperturbative effects. As well-known, the minimal topological charge $Q=1 /(k N)$ is achieved on torons [11] provided 't Hooft's twisted boundary conditions are imposed. The very possibility of imposing these boundary conditions rests on the fact the gauge group is $\mathrm{SU}(k N) / Z_{k N}$ rather than $\mathrm{SU}(k N)$ - the elements of the group center do not act on the adjoint fields. Equation (11) shows that two fermion zero modes exist in the toron background. These are so-called supersymmetric zero modes, $\lambda_{(0) \alpha} \propto F_{\alpha \beta}$, which are spatially constant. These modes saturate the gluino condensate $\langle\lambda \lambda\rangle$. In fact, calculation of the gluino condensate on $T^{4}$ along these lines was performed long ago [12].

If one introduces fields in the fundamental representation, the twisted boundary conditions become impossible since the elements of the center act nontrivially. However, the daughter theory with which we deal contains bifundamentals, not fundamentals. This fact allows one to resurrect twisted boundary conditions [13]. For simplicity we will discuss $Z_{2}$ orbifoldization, $k=2$. The case $k>2$ can be treated in a similar manner. For $k=2$ the daughter theory has two $\mathrm{SU}(N)$ groups, call them $\mathrm{SU}(N)^{\text {one }}$ and $\mathrm{SU}(N)^{\text {two }}$. The following boundary conditions are imposed [13] on the matter fields:

$$
\chi_{1,2}\left(L_{1}, y, z, t\right)=\Omega_{1}^{\mathrm{one}}(y, z, t) \chi_{1,2}(0, y, z, t) \Omega_{1}^{\mathrm{two}}(y, z, t)^{-1},
$$

\footnotetext{
${ }^{1}$ For the daughter theory this statement requires a reservation. In fact, $\mathcal{E}_{D}$ in Eq. (22) is a linear in $m$ part of the vacuum energy density. Unlike the parent supersymmetric theory, we cannot rule out a (nonperturbative) gluon condensate in the daughter theory. It may well exist, implying that the vacuum energy density has an $O\left(\mathrm{~m}^{0}\right)$ part at the nonperturbative level. In view of our negative conclusion on the NPO hypothesis it is only natural to assume that $\mathcal{E}_{D}$ has a nonvanishing $O\left(m^{0}\right)$ part.
} 


$$
\chi_{1,2}\left(x, L_{2}, z, t\right)=\Omega_{2}^{\mathrm{one}}(x, z, t) \chi_{1,2}(x, 0, z, t) \Omega_{2}^{\mathrm{two}}(x, z, t)^{-1}
$$

and so on, with one and the same twist in both $\mathrm{SU}(N)$ groups,

$$
\Omega_{\mu}^{\text {one, two }}\left(L_{\nu}\right) \Omega_{\nu}^{\text {one, two }}(0)=\Omega_{\nu}^{\text {one, two }}\left(L_{\mu}\right) \Omega_{\mu}^{\text {one, two }}(0) Z_{\mu \nu}
$$

where $Z_{\mu \nu}$ is an element of the $\mathrm{SU}(N)$ group center. This fact - that the twist is one and the same - follows from the single-valuedness of the fermion field.

The above boundary condition excludes field configurations with a toron in one of two $\mathrm{SU}(N)$ 's and topologically trivial gauge field in another. In other words, the minimal nonvanishing topological charge is associated with two torons, one in each $\mathrm{SU}(N)$. This automatically guarantees $Z_{2}$ symmetry. Equation (12) then implies that there are four fermion zero modes in this background. The bilinear condensate $\left\langle\chi_{1,2} \chi_{2,1}\right\rangle$ is not generated. Although the operator $\chi_{1,2} \chi_{2,1}$ is the orbifold partner to $\lambda \lambda$, there is no partner to the gluino condensate.

\subsection{Theory on $R^{3} \times S^{1}$}

In this subsection we discuss the theory on $R^{3} \times S^{1}$ world sheet (to be referred to as the theory on cylinder). In this case the gluino condensate was calculated [14] by saturating $\lambda \lambda$ by the "monopole instanton" configuration. Let us recall a few basic facts regarding the theory on $R^{3} \times S^{1}$. For definiteness we will take the fourth direction to be $S^{1}$.

Unlike the theory on $R^{4}$, where the appropriate topological classification is based on $\pi_{3}(\mathrm{SU}(k N))$, the situation is more complex for the theory on cylinder. The vacuum state is continuously degenerate (at the classical level) and is parametrized by the Polyakov line

$$
P \exp \left(i \int_{0}^{L} d t A_{4}(x, y, z, t)\right)
$$

which, upon diagonalization, can be represented as

$$
\exp \left(i \int_{0}^{L} a^{p} T^{p}\right)
$$

where $T^{p}$ are the generators of the Cartan subalgebra. The classically flat directions are parametrized by $k N-1$ moduli $a^{p}$ which are angular variables. The classical degeneracy of the vacuum manifold is lifted by nonperturbative effects [14] leading to $k N$ discrete vacua. The crucial point is that the moduli dynamics is $Z_{k N}$ invariant. This is again a consequence of the existence of the group center acting trivially on the adjoint fields. The $Z_{k N}$ invariance is clearly seen [14] in the superpotential generated on the moduli space through one "monopole instanton" saturation (more specifically, for $\mathrm{SU}(2)$, the prime target of Ref. [14]), it is $Z_{2}$ ). For the occurrence of this $Z_{k N}$ it is crucial that, along with the standard monopoles, the so-called KK monopoles are included in the calculation (for details see [14]). The latter are obtained from 
the standard monopoles by performing an improper gauge transformation - with a gauge matrix $\Omega(t)$ which is not periodic, $\Omega(t=L)=\Omega(t=0) Z$ where $Z$ is an element of the center. In the parent theory where all fields are in the adjoint, the inclusion of the KK monopole-instanton is automatic.

The "monopole instanton" action in the parent theory (in the supersymmetric vacuum) is

$$
A_{P}=\frac{8 \pi^{2}}{(k N) g_{P}^{2}}
$$

(see [14]). The "monopole instanton" has two gluino zero modes, which can be seen in many different ways. One can use, for instance, the fact that the "monopole instanton" has four bosonic zero modes. Supersymmetry then implies that the number of the fermion zero modes is two.

Since $k g_{P}^{2}=g_{D}^{2}$, the NPO matching requires that the action of the nonperturbative configuration relevant in the daughter theory is

$$
A_{D}=\frac{8 \pi^{2}}{N g_{D}^{2}} .
$$

This is the action of a single "monopole instanton" residing in one of the $\mathrm{SU}(N)$ factors of the daughter gauge group.

With respect to such field configuration, with the background field in just one $\mathrm{SU}(N)$, the daughter bifundamental fermion is nothing but an ensemble of $N$ flavors of fundamental fermions. For fundamental fermions the zero modes follow from the Callias index theorem [15, 16]. Say, at $k=2$ the four-dimensional Dirac operator $\not D_{4}$ can be considered. We look for $x_{4}$-independent zero modes. One can calculate the corresponding index and obtain that there is one zero mode for each field $\chi_{\ell, \ell+1}$ with one of the color indices lying in the given $\mathrm{SU}(N)$ - altogether $2 N$ modes.

Alternatively, instead of considering the Callias index, one can directly analyze the fermion zero modes in the "monopole instanton" background. As well-known for the 't Hooft-Polyakov monopole, with fundamental fermions coupled to the adjoint scalar field $\bar{\psi} \phi \psi$, one fermion zero mode per each flavor $\psi$ exists. The only distinction between the "monopole instanton" of Ref. [14 and the 't Hooft-Polyakov monopole is a slightly different chiral structure of the vertex coupling fermions to $\phi$ (or $A_{4}$ ). It is not difficult to see that this distinction has no impact on the existence of the fermion zero mode.

Summarizing, we conclude that while in the parent theory the "monopole instanton" has two fermion zero modes, its counterpart in the daughter theory has a large number of fermion zero modes, scaling with $N$. The corresponding condensates cannot match. 


\section{Orbifoldization of multiflavor theories and pat- terns of chiral symmetry breaking}

In this section we will consider parent-daughter pairs for multiple flavors. Both theories, the original one and the one obtained after orbifoldization, are non-supersymmetric. For simplicity we will focus on $Z_{2}$ orbifoldization and two flavors; $Z_{k}$ orbifoldization and $\nu$ "flavors" (with $k>2$ and $\nu>2$ ) can be analyzed in a similar manner.

Let the parent theory have $\mathrm{SU}(2 N)$ gauge group, and two Weyl flavors in the adjoint,

$$
\mathcal{L}=-\frac{1}{4} F_{\mu \nu}^{a} F^{\mu \nu, a}+\sum_{f=1}^{2}\left\{\bar{\lambda}_{\dot{\alpha},(f)}^{a} i \mathcal{D}^{\dot{\alpha} \alpha} \lambda_{\alpha,(f)}^{a}-m\left(\lambda_{(1)} \lambda_{(2)}+\text { h.c. }\right)\right\}
$$

where we find it convenient to change the overall normalization of fields compared to Eq. (4). The flavor index is indicated in parentheses. The mass term could be chosen in many equivalent forms, our choice is motivated by historical reasons (see [17]). We need the mass term for an auxiliary reason, to normalize the chiral condensates. Our final answer will refer to the limit $m \rightarrow 0$.

The orbifold theory is similar to Eq. (5), except that summation in the fermion sector runs over two flavors,

$\mathcal{L}=\sum_{\ell=1}^{2}\left\{-\frac{1}{4} F_{(\ell) \mu \nu} F_{(\ell)}^{\mu \nu}+\sum_{f=1}^{2} \bar{\chi}_{\ell, \ell+1,(f)} i \mathcal{D} \chi_{\ell, \ell+1,(f)}-m\left(\chi_{\ell, \ell+1,(1)} \chi_{\ell+1, \ell+2,(2)}+\right.\right.$ h.c. $\left.)\right\}$

(remember, $\chi_{2,3} \equiv \chi_{2,1}$ ). In the limit $m \rightarrow 0$ these two theories have flavor symmetries which are partially spontaneously broken resulting in the emergence of the Goldstone mesons. The pattern of the chiral symmetry breaking has been studied previously [17, 18. In the parent theory the flavor (non-anomalous) symmetry is $\mathrm{SU}(2)$. It is spontaneously broken down to $\mathrm{U}(1)$. Correspondingly, there are two Goldstones. The daughter theory is in fact (almost) QCD with two (Dirac) flavors. The non-anomalous flavor symmetry is $\mathrm{SU}(2) \times \mathrm{SU}(2) \times \mathrm{U}(1)$, which is spontaneously broken down to a diagonal $\mathrm{SU}(2)$ (the baryon number is also unbroken). Three familiar Goldstone bosons emerge. Thus, the dimensions of the vacuum manifolds in the parent and daughter theories (at $m=0$ ) are different.

A nonvanishing mass term discretizes the set of vacua, so that one can compare partner correlation functions in the parent/daughter theories in the vacuum with one and the same symmetry (i.e. $Z_{2}$ symmetric). We will consider Goldstones, derive analogs of the Gell-Mann-Oakes-Renner relations and show that, contrary to the NPO hypothesis, there is a mismatch in the chiral condensates in these two theories.

At first let us select a pair of corresponding and properly normalized currents in the parent/daughter theories producing Goldstone bosons. A convenient choice is

$$
J_{\alpha \dot{\beta}}^{P}=-\lambda_{\alpha,(1)} \bar{\lambda}_{\dot{\beta},(2)} .
$$


It is easy to see that in the daughter theory the current producing the same correlation function in the planar limit is

$$
J_{\alpha \dot{\beta}}^{D}=-\sqrt{2} \sum_{\ell} \chi_{\alpha,(1)} \bar{\chi}_{\dot{\beta},(2)} .
$$

Note a crucial overall $\sqrt{2}$ normalization factor in Eq. (33) which is absent in Eq. (32). It reflects $Z_{2}$ orbifoldization. At small momentum transfer, $p \ll \Lambda$, both correlation functions $\left\langle J^{P, D}(p), J^{\dagger P, D}(-p)\right\rangle$ have the form

$$
f^{2} \frac{p_{\mu} p_{\nu}}{p^{2}}
$$

with one and the same residue $f^{2}$ (in the limit $m \rightarrow 0$ ). The pole form is a direct consequence of the Goldstone saturation at $p \rightarrow 0$ while the equality of the residues is due to the NPO hypothesis.

Now, it is rather trivial to obtain the Gell-Mann-Oakes-Renner relations,

$$
\begin{array}{ll}
\text { Parent: } & \frac{f^{2} M^{2}}{m}=\left\langle 2\left(\lambda_{(1)} \lambda_{(2)}+\text { h.c. }\right)\right\rangle, \\
\text { Daughter: } & \frac{1}{2} \frac{f^{2} M^{2}}{m}=\left\langle 2 \sum_{\ell}\left(\chi_{(1)} \chi_{(2)}+\text { h.c. }\right)\right\rangle,
\end{array}
$$

where $M$ is the mass of the Goldstone boson while $m$ is the mass parameter in the Lagrangian, see Eqs. (30) and (31). Note that the ratio $M^{2} / m$ does not vanish in the limit $m \rightarrow 0$. Thus, both sides of the Gell-Mann-Oakes-Renner relations above are $O\left(m^{0}\right)$.

Now, the right-hand sides have to match since they present the slopes of the linear in $m$ part of the vacuum energy density. The left-hand sides do not match, however, because of the extra $1 / 2$ in the daughter theory, a leftover from $Z_{2}$ orbifoldization. Again, we conclude that the NPO conjecture implies a mismatch and thus cannot hold in the given parent/daughter pair.

\section{Comment on the brane picture}

A remark is in order regarding the observed mismatch of zero modes in weekly coupled theory on $R^{3} \times S^{1}$. How can one interpret it in the brane picture? In the brane picture the starting point for the parent $\mathrm{SU}(k N)$ theory is $k N \mathrm{D} 3$ branes wrapped around the compact direction. After the $T$ duality transformation they become D2 branes localized at the positions determined by the eigenvalues of the Wilson loop. The "monopole instanton" configurations discussed in Sec. 3.4 correspond to a Euclidean D0 brane stretched between two D2 branes. If we consider a $Z_{k}$ symmetric solution in the daughter theory then we have to take Euclidean D0 branes together with their $Z_{k}$ images; then only a multifermion (rather than bifermion) condensate 
can develop. If we count zero modes on the single D0 brane the mismatch is also evident since the number of zero modes in the daughter theory is larger due to additional branes representing bifundamentals.

\section{Conclusion}

The NPO hypothesis, if true, could be the most powerful tool for non-Abelian theories at strong coupling discovered in the last decade. Alas, the evidence we present in this paper suggests that this beautiful conjecture is not valid. The key question is: "can one modify this conjecture appropriately to make it work?"

The authors are grateful to Valya Khoze and Matt Strassler for useful discussions. The work of A.G. is supported in part by grants INTAS-00-334, CRDF-RP1-2108 and DE-FG02-94ER408. The work of M.S. was supported in part by DOE grant DE-FG02-94ER408.

\section{References}

[1] S. Kachru and E. Silverstein, Phys. Rev. Lett. 80, 4855 (1998) hep-th/9802183.

[2] A. E. Lawrence, N. Nekrasov and C. Vafa, Nucl. Phys. B 533, 199 (1998) hep-th/9803015.

[3] M. Bershadsky, Z. Kakushadze and C. Vafa, Nucl. Phys. B 523, 59 (1998) hep-th/9803076.

[4] M. Bershadsky and A. Johansen, Nucl. Phys. B 536, 141 (1998) hepth/9803249].

[5] M. Schmaltz, Phys. Rev. D 59, 105018 (1999) hep-th/9805218.

[6] M. R. Douglas and G. W. Moore, D-branes, Quivers, and ALE Instantons, hep-th/9603167.

[7] J. Lykken, E. Poppitz and S. P. Trivedi, Phys. Lett. B 416, 286 (1998) hepth/9708134;

J. Erlich and A. Naqvi, Nonperturbative tests of the parent/orbifold correspondence in supersymmetric gauge theories, hep-th/9808026.

[8] S. L. Dubovsky, Phys. Lett. B 492, 369 (2000) hep-th/0001186].

[9] M. J. Strassler, On methods for extracting exact non-perturbative results in non-supersymmetric gauge theories, hep-th/0104032.

[10] A. A. Belavin, A. M. Polyakov, A. S. Shwartz and Y. S. Tyupkin, Phys. Lett. B 59, 85 (1975). 
[11] G. 't Hooft, Commun. Math. Phys. 81, 267 (1981).

[12] E. Cohen and C. Gomez, Phys. Rev. Lett. 52, 237 (1984).

[13] E. Cohen and C. Gomez, Confinement And Chiral Symmetry Breaking With Twisted Gauge Configurations, HUTP-83-A026 (unpublished).

[14] N. M. Davies, T. J. Hollowood, V. V. Khoze and M. P. Mattis, Nucl. Phys. B 559, 123 (1999) hep-th/9905015;

N. M. Davies, T. J. Hollowood and V. V. Khoze, Monopoles, affine algebras and the gluino condensate, hep-th/0006011.

[15] C. Callias, Commun. Math. Phys. 62, 213 (1978).

[16] J. de Boer, K. Hori and Y. Oz, Nucl. Phys. B 500, 163 (1997) hep-th/9703100.

[17] Y. I. Kogan, M. A. Shifman and M. I. Vysotsky, Yad. Fiz. 42, 504 (1985) [Sov. J. Nucl. Phys. 42, 318 (1985)].

[18] D. Toublan and J. J. Verbaarschot, Nucl. Phys. B 560, 259 (1999) hepth/9904199]; J. J. Verbaarschot and T. Wettig, Ann. Rev. Nucl. Part. Sci. 50, 343 (2000) hep-ph/0003017. 